\title{
RESET
}

Recherches en sciences sociales sur Internet

$10 \mid 2021$

Savoirs incertains

\section{" Ils sont terrifiés par la médecine occidentale »}

Analyse des cadres accusatoires dans les médias sociaux entourant les pratiques funéraires traditionnelles lors d'Ebola

"They're terrified by Western medicine ". An analysis of accusatory frames in social media posts about traditional burial practices during Ebola

\section{Mélissa Roy}

\section{(2) OpenEdition}

\section{Journals}

Édition électronique

URL : https://journals.openedition.org/reset/3190

DOI : 10.4000/reset.3190

ISSN : 2264-6221

Éditeur

Association Recherches en sciences sociales sur Internet

Référence électronique

Mélissa Roy, « « Ils sont terrifiés par la médecine occidentale » », RESET [En ligne], 10 | 2021, mis en ligne le 13 mai 2021, consulté le 22 mai 2021. URL : http://journals.openedition.org/reset/3190 ; DOI : https://doi.org/10.4000/reset.3190

Ce document a été généré automatiquement le 22 mai 2021.

(c) Association Recherches en sciences sociales sur Internet 


\section{« Ils sont terrifiés par la médecine occidentale »}

Analyse des cadres accusatoires dans les médias sociaux entourant les pratiques funéraires traditionnelles lors d'Ebola

"They're terrified by Western medicine ". An analysis of accusatory frames in social media posts about traditional burial practices during Ebola

\section{Mélissa Roy}

\section{Introduction}

1 Lors d'éclosions de maladies contagieuses et infectieuses, la peur de la contagion affecte des rapports interpersonnels, pendant que des rationalités gestionnaires sont appliquées à des pratiques porteuses de sens et de lien social (Alcabes, 2009; Richard, 2016; Wald, 2008). Ainsi, lors de la pandémie actuelle de la COVID-19, le port obligatoire de masques, la distanciation physique et l'interdiction de rassemblements perturbent les salutations, fréquentations et habitudes sociales quotidiennes. Rappelons aussi l'enfermement généralisé et la modification des traditions funéraires religieuses lors de la peste, la « protection » des rapports sexuels motivée par la propagation de maladies sexuellement transmises, ou la fermeture d'écoles et de camps d'été pendant l'épidémie du virus H1N1 (Alcabes, 2009; Fabre, 1998; Hewlett \& Hewlett, 2008; Richards, 2016). La présente étude s'intéresse aux discours accusatoires qui émergent dans l'opposition entre une politique biosécuritaire ${ }^{1}$ et des socialisations et routines signifiantes sur le plan culturel. Elle prend comme cas d'étude l'éclosion d'Ebola (2013-2016), lors de laquelle des funérailles traditionnelles en Afrique de l'Ouest devinrent "sécurisées ». Elle propose d'analyser les discours circulant sur Twitter et Facebook qui accusent les rites funéraires d'être responsables de la diffusion de cette maladie. Cette recherche poursuit une étude antérieure (Roy et al., 2020), dans laquelle nous avons exploré le type de groupes sociaux blâmés pour l'épidémie Ebola sur ces médias sociaux: il sera ici 
question d'analyser les logiques argumentatives sous-jacentes au blâme afin d'explorer des dynamiques sociales à partir desquelles se déploient les accusations.

\section{Épidémies et accusations}

2 Le blâme, compris comme l'acte d'émettre un jugement de culpabilité, est une réaction récurrente dans l'histoire des épidémies de maladies contagieuses (Fabre, 1998; Farmer, 2006; McNeill, 1998; Nelkin \& Gilman, 1991). L'épidémie récente (2013-2016) de la maladie à virus Ebola a elle aussi donné lieu à son lot d'accusations : une partie des communautés africaines affectées par Ebola a été accusée, dans la couverture médiatique occidentale de l'épidémie, d'avoir contribué à la propagation de la maladie par leurs "pratiques culturelles", notamment la consommation de la viande de brousse et les rites funéraires traditionnels (Broom \& Broom, 2017; Mitman, 2014; Monson, 2017; Sinha \& Parmet, 2016). Certains gouvernements nationaux ont également été accusés d'un manque de préparation (Roy et al., 2020), et les "Blancs » ont été blâmés par des communautés en Afrique de l'Ouest pour leur prétendue participation volontaire à la propagation de la maladie (Landry Faye, 2015).

3 À l'instar de Douglas (1992), nous concevons l'accusation comme un commentaire social. En effet, un individu ou un groupe n'est pas uniquement blâmé pour l'événement indésirable duquel on le tient explicitement coupable : il est aussi situé dans un récit accusatoire plus large qui rappelle des actes répréhensibles qu'il aurait antérieurement commis (Dry \& Leach, 2010). Par conséquent, la figure blâmée devient un vecteur culturel dans lequel sont canalisés des anxiétés, espoirs, cadres et préjugés qui peuvent ne pas être directement liés à la situation présente (Farmer, 2006; Pascual, 2017). À cet égard, certains groupes se trouvent perpétuellement accusés pour divers événements et deviennent les boucs émissaires d'une communauté (Girard, 1982).

\section{Funérailles et Ebola}

4 Dans les trois pays principalement affectés par l'épidémie Ebola 2013-2016, la Guinée, le Libéria et la Sierra Leone, des croyances et pratiques animistes traditionnelles coexistent avec des religions du livre, dont l'islam et le christianisme (Marshall, 2017). En dépit de cette diversité religieuse, et sans renier la complexité des coutumes ni leurs particularités régionales, les rites funéraires traditionnels typiques ont tout de même des points communs importants. Les leaders religieux se chargent des funérailles et ont pour habitude de procéder au lavage du corps, à son habillement et à son maquillage. Le corps est ensuite exposé et fait l'objet de caresses, avant d'être enterré (Bagcchi, 2014; Faye et al., 2015; Piot, Muyembe \& Edmunds, 2014). Ces rites occupent un rôle important pour la famille et la communauté endeuillées car ils permettent de préparer le voyage du défunt dans l'autre monde. Inversement, des funérailles inappropriées condamnent son âme à errer (Fairhead, 2016).

5 Ce maniement des morts a été l'objet de préoccupations lors de l'épidémie Ebola (Green, 2014; Victory et al., 2015) parce que cette maladie se transmet par un contact direct avec les fluides corporels d'un corps symptomatique (Organisation mondiale de la santé [OMS], 2018). Le moment après la mort est particulièrement contagieux puisque la charge virale augmente au fil de la progression de la maladie (OMS, 2017). Ces événements traditionnels ont aussi été jugés inquiétants puisque de nombreuses 
personnes internes et externes au village assistent habituellement aux funérailles, ce qui peut propager la maladie à l'extérieur de la région affectée. Du mois de juin 2014 jusqu'à la mi-août 2014, les funérailles traditionnelles étaient la quatrième plus importante modalité de transmission d'Ebola, propageant environ 9\% des infections (Chowell \& Viboud, 2015). Par conséquent, un protocole d'« enterrements dignes et sécurisés "(EDS) a été implémenté dans les pays affectés. Ces EDS, incluaient l'aménagement de rituels funéraires dits sécurisés conduits par des professionnels vêtus d'équipement de protection individuelle et la désinfection des lieux où résidait le défunt (Le Marcis, 2015; Marshall \& Smith, 2015; Pandey et al., 2014).

6 La littérature scientifique sur les funérailles traditionnelles lors de l'épidémie Ebola se divise en deux groupes qui adoptent des positions thanatologiques antagonistes. Pour commencer, un corpus ancré dans une perspective sanitaire conçoit les funérailles traditionnelles comme des pratiques à risque de propager la maladie (Brainard et al., 2016; Curran et al., 2016; Omonzejele, 2014), et a pour double objectif de sensibiliser le public aux facteurs de risques d'infection et d'appeler à une sécurisation des pratiques funéraires «dangereuses » (Agua-Agum et al., 2016; Baştuğ \& Bodur, 2015; Cleaton et al., 2016; Jalloh et al., 2017). Un deuxième corpus, celui-ci socio-anthropologique, souligne les rôles sociaux remplis par les funérailles traditionnelles et critique l'implémentation de funérailles sécurisées, en ce que les autorités auraient insuffisamment considéré les perceptions locales (Chandler et al., 2015; Moulin, 2015; Ravi \& Gauldin, 2014). Dans ce contexte de tensions entre deux postures, plusieurs auteurs (Le Marcis, 2015; Richards, 2016) ont exploré, par le biais d'enquêtes ethnographiques, les conflits in situ entre la perspective sanitaire et celle socioanthropologique, et les accusations déployées de chaque côté. Cet article analysera donc un corpus de données différent, composé de commentaires publiés sur les médias sociaux.

7 Les médias sociaux sont un espace polémique, où circulent tant les discours officiels d'autorités sanitaires (Thackeray et al., 2012; Wong et al., 2017) - parfois (re)mis en circulation par des internautes (Chew \& Eysenbach, 2010; Fung et al., 2016; Nagpal et al., 2015; Odlum \& Yoon, 2015) - que les discours de résistance, tels que des rumeurs ou des «théories du complot » qui contredisent ou accusent les autorités sanitaires (Altani-Duault et al., 2015; Renard, 2011). Ainsi, les réseaux sociaux sont des arènes d'expressions et de controverses, où circulent de multiples interprétations d'un événement (Moreau et al., 2021; Picard et Vial, 2012; Vance, Howe \& Dellavalle, 2005; Yuya et al., 2012).

\section{Les cadres accusatoires}

8 Les études sur les accusations en temps d'épidémie tendent à recenser les groupes accusés de l'éclosion d'une maladie, et montrer en quoi ce blâme perpétue des dynamiques discriminatoires (Adeyanju, 2010; Mayor et al., 2013; Wagner-Egger et al., 2011). Nous voulons prolonger ces analyses, en nous intéressant de façon complémentaire aux logiques argumentatives qui justifient le déploiement d'accusations. Pour ce faire, nous utilisons le concept de "cadre» comme outil analytique. Lorsque compris dans une perspective d'analyse rhétorique des discours, le cadre renvoie à une interprétation structurée et partagée, qui investit du sens dans un événement (D’Angelo \& Kuypers, 2010; Kyriakides, 2017). Ce concept souligne l'existence de grands schèmes qui systématisent les significations investies dans un 
enjeu, et qui permettent d'interpréter ses causes, d'y émettre un jugement moral, et d'envisager des solutions particulières pour le gérer. En appliquant ce concept au blâme, nous postulons que les accusations prennent appui sur des schèmes plus généraux qui dépassent la situation pour laquelle on tient un individu ou groupe responsable, que nous nommons les cadres accusatoires. L'analyse des cadres accusatoires nous apparait pertinente puisqu'elle permet non seulement d'explorer les acteurs et groupes blâmés, mais aussi d'analyser les normes plus larges à partir desquelles se déploie l'accusation.

\section{Objectifs de recherche}

9 Cette recherche veut analyser, de façon inductive, les cadres accusatoires rattachés aux funérailles traditionnelles lors d'Ebola qui circulent sur Twitter et Facebook, afin de réfléchir aux dynamiques sociales qui servent de point d'ancrage à ce blâme. Il sera donc question de: 1) recenser les cadres accusatoires mobilisés; 2) explorer les façons dont ils sont utilisés pour blâmer certains groupes; 3) explorer les "autorités épistémiques » dont les propos sont remis en circulation pour servir de point d'appui aux arguments accusatoires; et 4) réfléchir aux dynamiques sous-jacentes aux cadres accusatoires déployés.

\section{Méthode}

10 Nous avons extrait des commentaires publiés par des usagers de médias sociaux sur Facebook et Twitter. Notre corpus de données a été construit en deux étapes. Pour commencer, nous avons récolté des tweets publiés en français ou en anglais, à partir d'une extraction en fonction de mots clés relatifs à Ebola et aux funérailles ["Ebola " ET (« funérai*» OU «funerals» OU « enterrement* » ou « burial* »)] ou à l'Ebola et à la religion [«Ebola» ET («religion» OU « Dieu» OU «God» OU «Allah» OU «prêtre* » OU «priest* " OU «pasteur* " OU «pastor* OU «imam*» OU «guérisseu*» OU «healer*»)]. Les mots associés à la religion ont permis de retenir des commentaires qui, sans aborder le mot clé "funérailles", parlaient de croyances religieuses, de leaders religieux et de spiritualité, des thématiques qui peuvent toutes concerner les funérailles. Le choix des mots-clés a été déterminé par les résultats d'une enquête préliminaire qui a mis en lumière les termes les plus employés pour discuter des funérailles traditionnelles sur Twitter et Facebook.

11 En raison de la coexistence de plusieurs religions dans les lieux affectés, une plus grande diversité de mots clés était employée pour aborder la thématique de la religion, en comparaison avec celle des funérailles. Ceci a donc mené à un dénombrement différent des mots clés employés pour les deux thématiques. Seuls les tweets publiés entre le $1^{\mathrm{er}}$ janvier 2014 (un mois après les premiers cas Ebola) et le 31 mars 2015 (deux mois après la déclaration de l'OMS qui annonçait que le nombre de nouveaux cas diminuait pour la première fois depuis le début de l'épidémie). Cette extraction a mené à un corpus de 235312 tweets, duquel un échantillon randomisé de 3469 tweets a été retenu ( $1,5 \%$ du corpus).

12 Le corpus de données inclut aussi des commentaires publiés via Facebook en réponse à des articles produits par 20 médias. Nous avons identifié les médias accessibles en ligne qui, selon l'index Alexa, sont les plus visités dans 10 pays, dont des pays de l'Afrique de 
l'Ouest affectés par Ebola (le Libéria, la Sierra Leone, la Guinée, le Nigéria, le Sénégal et le Mali) et un pays de l'Afrique de l'Ouest non-affecté par Ebola (la Côte d'Ivoire). Nous avons aussi choisi des médias de pays outre l'Afrique de l'Ouest puisque notre but est d'analyser des commentaires internationaux sur les funérailles, émis par des usagers géographiquement "près » de l'épidémie mais aussi par d'autres, «lointains ", afin de considérer les variations et points de recoupement dans les discours accusatoires. Ce faisant, nous avons identifié des médias dans trois pays européens et nord-américains (États-Unis, Canada et France). Nous avons sélectionné ces pays parce que leurs sites web avaient la plus grande quantité d'échanges sur la thématique des funérailles lors d'Ebola en français et en anglais, les deux langues principales dans les pays affectés en Afrique de l'Ouest.

13 Nous avons aussi identifié deux médias transnationaux qui sont consultés dans des pays africains, européens et nord-américains. Ensuite, nous avons extrait les articles publiés sur Facebook par ces médias entre les dates susmentionnées et contenant les mots-clés employés pour la récolte des tweets. Finalement, nous avons récolté un corpus de 1794 commentaires Facebook qui réagissaient à ces articles, en excluant ceux constitués de trois mots ou moins.

De cet échantillon de 5263 tweets et commentaires Facebook, 2489 commentaires ont été rejetés: 1648 utilisaient un mot-clé d'une façon non pertinente pour notre étude des cadres accusatoires (par exemple, en priant pour une amélioration de l'épidémie Ebola); 741 utilisaient un mot-clé hors contexte (par exemple, «Oh mon Dieu!»); 71 commentaires étaient des doublons; et 29 commentaires étaient insuffisamment détaillés pour être analysés. Ainsi, 2774 commentaires ont été analysés.

Les données extraites ont été divisées en deux groupes, chacun soumis à une analyse séparée. Le premier, les «données primaires ", inclut les commentaires publiés sur les médias sociaux qui partagent une opinion explicite et directe, dont l'analyse permet d'explorer les points de vue des usagers des médias sociaux analysés. Le second corpus, les «données d'escortes" (Simon \& Toullec, 2017), renvoie aux commentaires qui partagent des hyperliens ou des informations factuelles, sans prise de position explicite. Ce type de données influence le paysage discursif en accroissant la visibilité d'un certain type d'information. Puisqu'une grande partie des données d'escortes partageait uniquement des titres d'articles de médias sans URL et que la plupart des hyperliens partagés étaient expirés et inaccessibles au moment de l'analyse des données, seuls le type d'URL partagé et le titre des articles furent analysés, comme l'ont d'ailleurs fait des études précédentes (Roy et al., sous presse). Si ce procédé a comme inconvénient d'omettre les nuances et explications présentes dans le corps de l'article, il nous a permis de considérer une plus grande quantité de données puisqu'au moment de l'analyse, le contenu de plusieurs articles était inaccessible sur le web, alors que leur titre demeurait accessible sur les médias sociaux.

Nous avons ensuite subdivisé ces deux groupes de données (les données primaires et les données d'escorte) afin que l'analyse puisse tenir compte de l'usage différencié des cadres accusatoires selon deux facteurs : la plateforme de médias sociaux et le lien géographique des usagers. Pour ce faire, nous avons premièrement séparé les tweets et les commentaires Facebook dans chacun des deux premiers groupes de données, afin de permettre une comparaison des discours qui circulent sur chaque plateforme. Nous avons deuxièmement entamé une comparaison qui tient compte de la nature internationale des commentaires. Puisque seuls les commentaires bruts ont été 
récoltés, une limite de notre analyse relève de notre incapacité à considérer les variations dans les cadres accusatoires selon la géolocalisation des usagers. Cependant, dans le cas des commentaires Facebook, notre connaissance des médias publiant l'article sur lequel commentaient les usagers peut nous indiquer un lien ou une focale géographique.

Nous avons donc analysé les données dans les deux premiers groupes de façon séparée, en distinguant: 1) les données indéterminées (tweets et commentaires Facebook répondant à des articles publiés par des médias transnationaux); 2) les commentaires Facebook rattachés à des articles publiés par des médias européens ou nord-américains; et 3) les commentaires Facebook répondant à des articles publiés par des médias de l'Afrique de l'Ouest.

Plus précisément, notre démarche analytique s'est inspirée de l'analyse rhétorique des cadres (D’Angelo \& Kuypers, 2010; Kyriakides, 2017) qui veut identifier les paramètres argumentatifs de définition d'un problème. Nous avons effectué une analyse qualitative manuelle dans le logiciel Nvivo, où chaque commentaire récolté a été associé à trois codes maximum. Le premier code renvoie aux cadres qui investissent des significations dans les funérailles traditionnelles. Le deuxième code relève des figures accusées de la propagation d'Ebola via les funérailles. Le troisième code a trait aux modalités de justification des accusations.

\section{Limites méthodologiques}

Les connaissances produites dans cet article sont limitées par certains éléments. Il nous est impossible de connaître la population d'enquête puisque les informations (genre, géolocalisation, appartenance culturelle, caractéristiques sociodémographiques) n'ont pas été récoltées pour des raisons techniques et éthiques. Néanmoins, celles-ci ne sont pas toujours disponibles puisque des informations ne sont pas publiques et certaines autres, comme la géolocalisation des comptes d'usagers de médias sociaux et la géolocalisation des commentaires publiés, ne donnent qu'un portrait partiel de l'appartenance nationale ou culturelle : par exemple, ce n'est pas parce qu'un compte ou un commentaire est associé à un certain pays que l'usager y habite ou en a la nationalité. Afin de développer une analyse nuancée, qui tienne compte de la population qui utilise les cadres accusatoires, il faudrait combiner l'analyse de données sur les médias sociaux à des questionnaires ou entretiens menés auprès des usagers qui publient les commentaires analysés.

De plus, notre intention initiale était d'analyser les articles de médias qui ont permis la collecte de commentaires Facebook, puisque leur contenu est susceptible d'influencer la teneur des commentaires Facebook retenus. Or, nous avons seulement pu retracer un petit nombre d'articles $(\mathrm{n}=82$, dont 38 avaient un contenu accusatoire pertinent pour notre étude). De plus, une plus grande proportion d'articles publiés par des médias en Afrique de l'Ouest était inaccessible au moment de l'analyse, en raison d'un manque de ressources (serveurs, etc.) qui ne permet pas d'assurer l'archivage des articles. Ainsi, en raison de cette petite quantité d'articles et de la surreprésentation de médias en Amérique du Nord et Europe, nous avons laissé de côté l'analyse des discours médiatiques. 


\section{Analyse}

21 Notre analyse indique que les funérailles traditionnelles sont surtout critiquées dans les échanges sur les médias sociaux $(\mathrm{n}=2 \quad 746,99 \%)$. Le débat thanatologique qui traverse la littérature scientifique et les discours in situ, ne s'est pas traduit sur les médias sociaux, où les commentaires analysés se situent presque exclusivement du côté sanitaire de la controverse. Ces accusations entourant les funérailles lors d'Ebola prennent appui sur cinq cadres (voir le tableau 1).

Tableau 1 - Synthèse des cadres accusatoires

\begin{tabular}{|c|c|c|c|c|}
\hline $\begin{array}{l}\text { Cadre } \\
\text { accusatoire }\end{array}$ & $\begin{array}{l}\text { Pourcentage } \\
\text { du corpus } \\
\text { concerné }\end{array}$ & Arguments & $\begin{array}{l}\text { Principales cibles } \\
\text { du blâme }\end{array}$ & $\begin{array}{l}\text { Pourcentage de } \\
\text { commentaires } \\
\text { visant chaque } \\
\text { cible (par cadre } \\
\text { accusatoire) }\end{array}$ \\
\hline \multirow[b]{2}{*}{$\begin{array}{l}\text { Opposition } \\
\text { religion-science }\end{array}$} & \multirow[b]{2}{*}{$47 \%$} & \multirow[b]{2}{*}{$\begin{array}{l}\text { Critique des } \\
\text { interprétations } \\
\text { spirituelles. } \\
\text { Étiquetage des croyances } \\
\text { religieuses de } \\
\text { "primitives" et } \\
\text { d' «irrationnelles». } \\
\text { Mise en opposition de la } \\
\text { religion à la science, la } \\
\text { médecine et la « Raison». }\end{array}$} & Leaders religieux & $84,7 \%$ \\
\hline & & & L'« Afrique» & $5,2 \%$ \\
\hline \multirow{3}{*}{$\begin{array}{l}\text { Inaction et } \\
\text { irresponsabilité }\end{array}$} & \multirow{3}{*}{$37,2 \%$} & \multirow{3}{*}{$\begin{array}{l}\text { Mobilise la question de } \\
\text { l'agentivité et du } \\
\text { «choix» de ne pas agir } \\
\text { « comme il le faut». } \\
\text { Critique l'action à l'écart } \\
\text { des prescriptions } \\
\text { sanitaires. } \\
\text { Présentation des lieux et } \\
\text { groupes affectés comme } \\
\text { «refusant de s'aider». } \\
\text { Justifie un } \\
\text { désenchantement à } \\
\text { l'égard de l'aide } \\
\text { humanitaire. }\end{array}$} & $\begin{array}{l}\text { Habitants des } \\
\text { lieux affectés }\end{array}$ & $81,5 \%$ \\
\hline & & & $\begin{array}{l}\text { Gouvernements } \\
\text { nationaux des } \\
\text { pays affectés par } \\
\text { Ebola }\end{array}$ & $15,4 \%$ \\
\hline & & & L' « Afrique » & $1,9 \%$ \\
\hline \multirow[b]{2}{*}{$\begin{array}{l}\text { Cultures } \\
\text { locales/ } \\
\text { africaines }\end{array}$} & \multirow[b]{2}{*}{$9,2 \%$} & $\begin{array}{l}\text { Conceptualisation de } \\
\text { l'épidémie comme le } \\
\text { produit de coutumes et } \\
\text { pratiques culturelles. }\end{array}$ & $\begin{array}{l}\text { Habitants des } \\
\text { lieux affectés }\end{array}$ & $45,3 \%$ \\
\hline & & $\begin{array}{l}\text { Généralisation des } \\
\text { coutumes à l' "Afrique ". } \\
\text { Association des pratiques } \\
\text { et croyances culturelles à }\end{array}$ & & \\
\hline RESET, 10 | 2021 & & $\begin{array}{l}\text { la "tradition", au } \\
\text { "primitif " et à } \\
\text { l' « irrationalité ». }\end{array}$ & & \\
\hline
\end{tabular}




\begin{tabular}{|c|c|c|c|c|}
\hline & & & L'« Afrique » & $12,5 \%$ \\
\hline La corruption & $4,7 \%$ & $\begin{array}{l}\text { Rarement détaillé. } \\
\text { Habituellement mobilisé } \\
\text { en complément, pour } \\
\text { renforcer la critique d'un }\end{array}$ & $\begin{array}{l}\text { Gouvernements } \\
\text { nationaux des } \\
\text { pays affectés par } \\
\text { Ebola }\end{array}$ & $57,6 \%$ \\
\hline & & $\begin{array}{l}\text { groupe accusé pour un } \\
\text { enjeu qui précède Ebola }\end{array}$ & Leaders religieux & $27,3 \%$ \\
\hline Pauvreté & $1,9 \%$ & $\begin{array}{l}\text { Blâme les lacunes dans } \\
\text { l'infrastructure sanitaire } \\
\text { des pays affectés. } \\
\text { Mise en opposition des } \\
\text { pays en Afrique de } \\
\text { l'Ouest affectés avec les } \\
\text { pays de l'Europe et de } \\
\text { l'Amérique du Nord }\end{array}$ & L'« Afrique » & $76,9 \%$ \\
\hline
\end{tabular}

Le premier cadre accusatoire émet une critique générale de toutes les religions présentes dans les lieux affectés (animistes, chrétienne et islamique) ( $n=327)$. Elles sont classées dans le champ de l'irrationalité et du primitif, et mises en opposition à la science et à la médecine, symboliques de la modernité. $47 \%$ des commentaires relève du cadre "opposition religion-science", et au sein de ce groupe, $84,7 \%$ vise les leaders religieux et $5,2 \%$ vise l'« Afrique ».

Le deuxième cadre accusatoire évoque l'«inaction » et l'«irresponsabilité » des figures blâmées $(\mathrm{n}=259)$. La question d'agentivité traverse ce cadre accusatoire, au travers duquel on souligne que des individus ou groupes connaissent les «bonnes pratiques » à mettre en oeuvre afin de gérer l'Ebola et d'éviter sa propagation, mais qu'ils choisissent de ne pas agir ainsi, par exemple en pratiquant les funérailles traditionnelles. Ce type d'accusation est classique dans le champ des épidémies puisque les éclosions de maladies sont rarement interprétées comme un événement aléatoire et plutôt perçues comme le produit de choix, de négligences et d'erreurs (Alcabes, 2009; McNeill, 1998).

Troisièmement, les accusations entourant les funérailles puisent dans un cadre qui aborde les «cultures locales ou africaines » $(n=64)$ qui affirme que la persistance des rites non sécurisés est le produit de coutumes, conçues comme des traditions primitives.

25 La corruption est un quatrième cadre accusatoire mobilisé sur Twitter et Facebook $(\mathrm{n}=33)$. Notons que celui-ci est rarement détaillé, habituellement mobilisé pour ajouter une critique additionnelle à l'encontre d'un groupe déjà accusé de la propagation d'Ebola. Ce cadre est un commentaire social qui dépasse l'épidémie puisqu'il permet de critiquer un enjeu qui précède Ebola, comme des leaders religieux qui mentent, manipulent la congrégation et sont mercantiles.

Finalement, le cadre de pauvreté $(n=13)$ blâme des lacunes dans l'infrastructure sanitaire des pays affectés, et sert à rassurer ceux qui habitent en Amérique du Nord en Europe, par le biais d'une comparaison qui met en exergue leur soi-disant bonne infrastructure sanitaire, « meilleure » que celle des lieux affectés. 


\section{Les figures du blâme} accusatoires sont mobilisés pour la critiquer. Elle serait trop ancrée dans la religion et insuffisamment dans la science: «À cause de la religion, beaucoup d'africains pensent qu'Ebola n'existe pas ou se guérit avec la prière. C'est triste." ${ }^{4}$. Elle serait "indocile», en raison de son refus d'agir "correctement » : "Les Africains seront peut-être fâchés quand ils l'entendront, mais ils doivent arrêter de toucher les corps morts pendant les funérailles. $\|^{5}$ Elle serait le lieu de "mauvaises » coutumes et d'une culture à changer : "Le principal problème qu'on a en \#Afrique, c'est les rites funéraires. Il y a des progrès à faire. $»^{6}$. Elle serait « corrompue »: "Complètement fou: l'Afrique est tellement à un stade de développement et de maturité de gouvernance primitif, en ce qui concerne la corruption, la transparence et l'imputabilité».'

31 Et, finalement, elle aurait un « mauvais » système de santé: « Les meilleurs soins médicaux, ne sont pas en Afrique. Ils sont aux États-Unis, où les meilleurs établissements de recherche du monde sont situés, et où le travail avec le virus actif a lieu depuis des années. $»^{8}$ L'ensemble de ces cadres accusatoires transforment l'«Afrique» en une "figure à problèmes", sur laquelle il convient d'intervenir afin de l'«éduquer », l'aider ou l'exclure, comme illustré par des commentaires tels que "Différences tribales, VIH, virus Ebola, le 'Prophète' ... Plusieurs raisons pour lesquelles l'Afrique a besoin de plus d'éducation et moins de superstition! 
$»^{9}$ ou "Il faut mettre un dôme géant par-dessus l'Afrique. Ces tribus ignorantes répéteront les mêmes pratiques jusqu'à ce qu'elles soient éteintes. $»^{10}$

\section{Les autorités épistémiques des cadres accusatoires} nommons des « autorités épistémiques » (Broudoux, 2017). Nous utilisons ce qualificatif pour désigner une personne, un groupe ou une institution qui a la légitimité de dire ce qui est. Nous avons constaté la présence de deux principales autorités épistémiques dans les cadres accusatoires mobilisés au sein des commentaires analysés: les institutions de santé publique globales et les médias. Les usagers de médias sociaux expriment un accord quant aux publications de ces institutions, partagent leurs discours et les remettent en circulation pour appuyer et justifier leurs propres arguments.

\section{Les discours sanitaires}

Pour commencer, notre analyse des données d'escorte montre que de nombreux usagers de médias sociaux (26,32\% des tweets, $25,12 \%$ des commentaires Facebook) citent des institutions de santé publique et globale, notamment l'OMS, et mobilisent des propos sanitaires «factuels» sur la maladie: «@OMS: Au moins 20\% des nouvelles infections \#Ebola ont lieu pendant les enterrements de patients Ebola décédés ${ }^{11}$. Certains usagers relaient aussi les discours émis par des institutions sanitaires qui soulignent la persistance de funérailles traditionnelles et l'efficacité des enterrements sécurisés, comme illustré par des commentaires tels que «Ebola: des enterrements secrets seraient pratiqués en Afrique de l'Ouest selon l'OMS $»^{12}$ et $~ " O M S$ : Les décès d'Ebola approchent les 5000, de nouvelles pratiques d'enterrement aident à diminuer la propagation ${ }^{13}$. Ce partage d'information sanitaire communique une méfiance à l'égard des funérailles qui alimente une critique des pratiques «culturelles» et des «traditions » locales : «Les rites funéraires : une tradition qui condamne les populations les plus touchées par le virus Ebola ... (Hyperlien du site de l'OMS) $»^{14}$ Ainsi, les usagers qui remettent en circulation cette information qui provient d'autorités sanitaires alimentent un paysage discursif accusatoire à l'encontre des habitants des lieux affectés.

\section{Les discours médiatiques}

Les médias sont aussi dotés d'une autorité épistémique dans les commentaires analysés. Pour commencer, nos résultats montrent que les discours véhiculés au sein d'articles de médias sont répétés dans les commentaires Facebook qui les commentent. En effet, des commentaires Facebook citent ou paraphrasent le contenu médiatique (« Les médias 'Main Stream', nous expliquent que cette épidémie progresse sur l'ignorance, le manque d'hygiène et les rites funéraires inadéquats... $\nu^{15}$ ), et expriment un accord avec le contenu d'un article ("C'est l'un des meilleurs articles que j'ai lu sur comment régler les problèmes au Libéria sous le présent gouvernement $\left.»{ }^{16}\right)$

Cette présence des discours médiatiques dans les accusations sur Facebook peut certes être le produit partiel de notre protocole de collecte de données, où les commentaires extraits de Facebook sont directement rattachés à des articles de médias. Or, cette autorité épistémique des médias est aussi perçue dans les tweets, qui n'étaient pourtant 
pas nécessairement rattachés à des articles de médias. En fait, les tweets analysés sont caractérisés par une plus grande remise en circulation du contenu médiatique brute, sans prise de position, en comparaison avec les commentaires Facebook. Cette différence entre Facebook et Twitter peut être expliquée par l'appropriation différenciée des deux plateformes étudiées par les usagers de médias sociaux. En effet, Twitter est souvent utilisé comme un outil de reportage via lequel de courts commentaires factuels sont publiés (140 signes, au moment de la recherche) et des hyperliens sont partagés (Oh, Agrawal et Raghay, 2013). Quant à Facebook, il est surtout employé comme espace de publication de sentiments et pensées en phrases ou paragraphes (Orchard et al., 2015). Nous résultats (voir le tableau 2) indique que la thématique est plus débattue sur Facebook et que les cadres accusatoires y sont plus fréquemment mobilisés, ce qui corrobore les résultats d'une étude précédente (Moreau et al., 2021) qui montre que des débats deviennent politisés sur cette plateforme.

Tableau 2 - Pourcentage d'arguments inscrits dans chaque cadre accusatoire selon le corpus de données

\begin{tabular}{|l|l|l|l|l|l|}
\hline & $\begin{array}{l}\text { Opposition } \\
\text { religion-science }\end{array}$ & $\begin{array}{l}\text { Inaction ou } \\
\text { irresponsabilité }\end{array}$ & $\begin{array}{l}\text { Cultures } \\
\text { locales/ } \\
\text { africaines }\end{array}$ & Corruption & Pauvreté \\
\hline $\begin{array}{l}\text { Commentaires } \\
\text { Facebook }\end{array}$ & $17,76 \%$ & $12,4 \%$ & $8,38 \%$ & $5,36 \%$ & $2,18 \%$ \\
\hline Tweets & $0,96 \%$ & $8,5 \%$ & $0,64 \%$ & $0,5 \%$ & 0 \\
\hline
\end{tabular}

Les histoires médiatiques les plus fréquemment partagées dans les tweets abordent la propagation d'Ebola lors des funérailles d'une guérisseuse traditionnelle qui aurait contracté la maladie en soignant un patient ( $\mathrm{n}=77)$, les habitants des lieux affectés qui attaquent des fossoyeurs $(\mathrm{n}=73)$ et les corps délaissés dans la rue par ces habitants $(n=11)$. Quoique ces histoires ne soient pas accusatoires en soi, elles contribuent à forger un paysage discursif qui souligne le rôle des mauvaises actions et mauvaises traditions des habitants des lieux affectés dans l'épidémie. Dans la mesure où ce type d'information devient très visible, très facilement accessible, il peut engendrer des accusations. Si une limite de notre étude est l'incapacité de déterminer l'impact des commentaires publiés par des usagers de médias sociaux dans le monde "réel», nos résultats mettent tout de même en lumière un risque potentiel de dynamiques accusatoires suscitées par la (re)mise en circulation de certaines histoires médiatiques.

Notons finalement que les discours médiatiques et sanitaires ne sont pas séparés. Une partie du contenu médiatique ne fait que transmettre l'information véhiculée par les autorités sanitaires. Plusieurs tweets, par exemple, partagent des articles de médias qui transmettent des données épidémiologiques sur le rôle des funérailles dans la propagation de la maladie : «Les Chercheurs unanimes : les rites funéraires à l'origine de la propagation du virus Ebola (Hyperlien vers un article de médias) ${ }^{17} \gg$ Le partage de ce type d'information communique une critique des "mauvais actes" et de la "culture " des habitants des lieux affectés, d'une façon telle que les dynamiques accusatoires influencées par les discours sanitaires sont aussi présentes dans les discours 
médiatiques. Ces deux autorités épistémiques opèrent donc parfois conjointement pour servir de point d'appui au paysage discursif accusatoire.

\section{La récurrence de la figure de l'« Afrique »} evoquée dans les données rattachées à des médias européens et nord-américains, enclins à généraliser et à dévaloriser ce continent. Or, cette dynamique est aussi présente dans les commentaires de contenus médiatiques africains (voir le tableau 4) et des usagers de médias sociaux qui se désignent eux-mêmes comme "Africains" critiquent l' "Afrique " et l' "Africain ", émettant ainsi une accusation auto-dirigée. «Ebola est principalement une menace pour les Africains car, contrairement aux autres races, nous refusons d'agir avec discernement. Nous ne faisons jamais les bonnes choses que nous savons faire. ${ }^{18}$ Or, ce cadre accusatoire est presque aussi présent dans les données rattachées à des médias d'Afrique de l'Ouest (voir le tableau 3).

Tableau 3 - Répartition des résultats selon le rapport géographique des commentaires

\begin{tabular}{|l|l|l|l|}
\hline & $\begin{array}{l}\text { Commentaires de médias du } \\
\text { Nord (France, États-Unis, } \\
\text { Canada) } \\
(\mathrm{n}=249)\end{array}$ & $\begin{array}{l}\text { Commentaires de médias africains } \\
\text { (Sierra Leone, Libéria, Guinée, Nigéria, } \\
\text { Mali, Sénégal, Côte d'Ivoire) } \\
(\mathrm{n}=310)\end{array}$ & Total \\
\hline
\end{tabular}




\begin{tabular}{|l|l|l|l|}
\hline Figures du blâme & & & \\
\hline Leaders religieux & $29(11,7 \%)$ & $48(15,5 \%)$ & 290 \\
\hline $\begin{array}{l}\text { Habitants des lieux } \\
\text { affectés }\end{array}$ & $18(7,2 \%)$ & $53(17,1 \%)$ & 279 \\
\hline $\begin{array}{l}\text { Gouvernements } \\
\text { nationaux }\end{array}$ & $31(12,5 \%)$ & $54(17,4 \%)$ & 93 \\
\hline «Afrique » & $21(8,4 \%)$ & $12(3,9 \%)$ & 40 \\
\hline $\begin{array}{l}\text { Cadres } \\
\text { accusatoires }\end{array}$ & $38(15,3 \%)$ & $58(18,7 \%)$ & 327 \\
\hline $\begin{array}{l}\text { Opposition } \\
\text { «religion-science » }\end{array}$ & $6(3,2 \%)$ & $51(16,5 \%)$ & 64 \\
\hline $\begin{array}{l}\text { Inaction } \\
\text { irresponsabilité }\end{array}$ & $20(8 \%)$ & $25(8,1 \%)$ & 259 \\
\hline $\begin{array}{l}\text { Cultures locales en } \\
\text { Afrique }\end{array}$ & $22(8,8 \%)$ & $5 \%)$ & 33 \\
\hline Corruption & $\begin{array}{l}\text { Pauvreté } \\
\text { (2,4\%) }\end{array}$ & & \\
\hline
\end{tabular}

41 L'ensemble de ces constats peut s'expliquer par le concept de « la fatigue des maladies infectieuses émergentes » de Joffe (2011). Selon l'auteure, la récurrence d'épidémies s'associe à la perception que la maladie est produite par l'humain et ainsi à la croissance d'une frustration auto-dirigée puisqu'on se perçoit - soi-même et les institutions qui nous représentent - comme responsables de cet enjeu réémergent. À cet égard, nous constatons une plus grande présence du cadre axé sur l'inaction et l'irresponsabilité dans les données rattachées aux médias en Afrique de l'Ouest en comparaison avec celles rattachées aux médias de l'Europe et d'Amérique du Nord (environ deux fois plus). Cet accent sur les "mauvais comportements» traduit la perception que l'épidémie est le produit de pratiques inadéquates «chez soi » qui perdurent.

Somme toute, la «fatigue des maladies infectieuses émergentes » en " Afrique » serait ici articulée par un « repli sur soi » (Roy et al., 2020) caractérisé par la critique de son « inaction » et « irresponsabilité » et de sa propre « race » et "culture », deux attributs ambigus sur lesquels les habitants des lieux affectés projettent les actes et enjeux récurrents qu'ils réfutent:

Plutôt que de commencer à mettre en œuvre des pratiques sécurisées, nous, Africains, nous emmêlons souvent dans la misère en raison de nos propres actions. Ebola a commencé en Afrique centrale et de l'Est il y a plusieurs années. [...] Depuis ce temps, qu'a fait que notre gouvernement pour essayer d'éliminer cette maladie? Les personnes continuent à acheter de la 'viande de brousse' qui est probablement sous-cuite parce que les vendeurs se dépêchent. [...] On ne peut pas continuer 
d'accuser le blanc pour tout ce qui nous arrive. Le SIDA auquel tu te réfères peut être arrêté si les personnes portent des préservatifs pendant la pénétration. [...] On prend nos décisions et on devrait en être tenus responsables. ${ }^{19}$ disent "Africains" s'inscrit dans un mécontentement qui dépasse l'épidémie Ebola. Entre autres, le VIH/SIDA est rappelé comme «faillite africaine » par des personnes qui ont un lien d'appartenance avec l'Afrique, qui interprètent des événements contemporains comme le prolongement d'enjeux qui ont amplifié le VIH dans cette région mondiale.

\section{Le cadre de l'irrationalité}

Une lecture transversale des divers cadres accusatoires illustre une critique du savoir profane, conçu comme «irrationnel ». En effet, les multiples cadres ont en commun de valoriser les acteurs " objectifs » et "rationnels ", "éclairés » par le savoir scientifique, et intégrés (par leurs discours et pratiques) dans l'épistémologie biomédicale d'Ebola. Ceux qui se distancient d'un récit purement positiviste, qui expriment des croyances religieuses, ne se conforment pas aux prescriptions d'autorités, apprécient ou respectent les coutumes, et sont influencés par des valeurs sociales plutôt que par les discours médicaux, sont objets d'accusation et classés dans le champ de l'irrationalité. Ainsi, l' «ignorance», le "refus de la médecine», les "mauvaises connaissances", l' « irrationalité », la «stupidité », l' « analphabétisme », l' « illettrisme » sont tous mobilisés comme schèmes explicatifs des comportements critiqués par des discours qui s'inscrivent dans les cadres accusatoires recensés :

La principale raison pour laquelle Ebola se répand est due à une population ignorante. Taux d'analphabétisme de 70\%. [...] La population est superstitieuse. J'ai entendu les choses les plus ridicules directement de la part des Libériens euxmêmes, y compris le fait que les hommes en blanc vaporisent du sperme d'Ebola au lieu de l'eau de Javel pour contaminer les gens. [...] Ils sont terrifiés par la médecine occidentale et croient qu'ils sont maudits. Ils se cachent donc dans leur maison et infectent toute leur famille. ${ }^{20}$

L'excès d'émotion sert aussi d'explication aux comportements critiqués par les cadres accusatoires : les cibles du blâme sont spirituelles, indociles, conformes à des coutumes, corrompues, etc. parce que «trop sentimentales", comme illustré par « ̀̀ quoi sert le fait d'être émotif et de rapporter un corps chez soi pour enterrement, seulement pour ensuite avoir une multiplication des enterrements à cause de la propagation rapide d'Ebola? $»^{21}$ ou $"$ Si on ne fait pas attention, on se retrouvera dans des situations difficiles parce qu'on aura laissé nos peurs contrôler notre intelligence. $»^{22}$

Cette valorisation de la «rationalité » affirme une supériorité de la "Science », élevée comme « valeur sociétale » (Ward, Guille-Escuret \& Alapetite, 2019). Ce type de discours peut s'avérer rassurant puisqu'il réduit l'épidémie à un problème "d'écart à la science ", d'une façon qui proclame la capacité mondiale à gérer les épidémies dans la mesure où « on n'est guidé que par la science ». Cette critique de l'«irrationalité » dans les accusations communique aussi la crainte d'une chute du pouvoir de la "vérité scientifique ", une préoccupation sociale répandue et qu'on témoigne dans les discours sur la "post-vérité » et la «désinformation» (Baggini, 2017; Hyvönen, 2018; KoroLjungberg, Carlson \& Montana, 2019; Paul \& Haddad, 2019; Prozorov, 2019). En effet, la popularisation contemporaine de ces deux concepts traduit une appréhension chez le grand public et chez les décideurs politiques, qui craignent une crise d' "irrationalité » 
diffuse où des faussetés atteignent le statut de vérité (Leroy et al., 2018). Dans notre étude, cette crainte du tissu de "post-vérité " et de "désinformation» se traduit par l'importance accordée à l' "irrationalité" pour justifier la critique des discours religieux, des résistances aux prescriptions des autorités sanitaires et des pratiques culturelles. Somme toute, cet accent sur l'écart à la raison réduit la tension récurrente entre la sécurisation et le maintien de liens sociaux à un problème d' "ignorance ", en omettant des interprétations plus nuancées axées sur l'importance des socialisations et significations culturelles dans les pratiques quotidiennes (Fabre, 1998; Richards, 2016).

\section{Conclusion}

Le présent article a analysé les cadres accusatoires mobilisés par des usagers de médias sociaux sur Twitter et Facebook pour commenter les funérailles lors de l'épidémie Ebola 2013-2016. Nos résultats indiquent que les usagers de ces médias sociaux accusent certains groupes (notamment les leaders religieux, les habitants des lieux affectés, l' «Afrique » et les gouvernements nationaux) de la propagation d'Ebola par l'entremise de funérailles traditionnelles. Cette accusation s'appuie sur des discours qui critiquent des pratiques et schèmes en les qualifiant $\mathrm{d}$ ' «irrationnels ». Ce faisant, les accusations entourant les funérailles traditionnelles au moment de l'épidémie de maladie à virus Ebola dépassent l'épidémie. En effet, nous émettons l'hypothèse que ce blâme prend appui dans la rhétorique de la "post-vérité ", qui met en scène la crainte de la désinformation. Les discours qui entourent l'enjeu de la "post-vérité » servent ici à réitérer des discours discriminants à l'encontre de « figures du blâme » (Atlani-Duault et al., 2015) récurrentes comme l' "Afrique». Notre recherche montre ainsi comment une étude généralisée des dynamiques en ligne pourrait mettre en lumière un racisme insidieux par lequel des figures racisées sont infériorisées par des discours qui n'évoquent pas explicitement leur « différence raciale ». Rappelons cependant que des usagers de médias sociaux qui se revendiquent comme "Africains» critiquent aussi l' «Afrique » et expriment une frustration auto-dirigée, parce qu'ils conçoivent l'Ebola comme le symptôme de problèmes récurrents. Ainsi, notre étude contribue aux travaux sur les accusations en temps d'épidémies en montrant comment le blâme réarticule des scripts sociaux préexistants (Farmer, 2006; Joffe, 2004) à partir de discours qui traversent déjà l'espace social (discours de post-vérité, discours de frustration). En résumé, quatre dynamiques alimentent les cadres accusatoires entourant les funérailles traditionnelles lors d'Ebola: la peur du virus, la peur de l'Autre, la peur de la désinformation, et la critique de dysfonctionnements institutionnels dont l'épidémie Ebola n'est qu'un symptôme.

Finalement, notre recherche montre une influence des autorités sanitaires et médiatiques dans ces accusations et illustre le potentiel d'influence de ces institutions, qui peuvent alimenter un paysage discursif accusatoire même si leurs publications ne blâment pas explicitement. En mettant l'accent sur des éléments comme la "culture " ou la «tradition » et en diffusant des articles qui n'offrent pas un portrait juste de l'importance sociale, culturelle et politique des pratiques funéraires (Fairhead, 2016), elles réduisent et simplifient la situation d'une façon qui peut ensuite être reprise pour justifier des discours accusatoires. Il est ainsi primordial que ces autorités épistémiques suivent leurs publications en ligne, en lisant les commentaires qui y réagissent et en 
explorant le nombre de partages, afin d'ajuster ensuite leurs publications pour nuancer les discours dominants qui peuvent être repris pour accuser.

\section{BIBLIOGRAPHIE}

\section{Références}

ADEYANJU Charles (2010). Deadly fever. Racism, disease and a media panic. Halifax, Fernwood.

AGUA-AgUm Junerlyn, ARIYARAJAH Archchun, AYLWARd Bruce, BAWo Luke, BILIVOGUI Pepe, BLAKE Isobel, ... YОTI Kabulon (2016). « Exposure patterns driving Ebola transmission in West Africa: A retrospective observational study ", PLoS Medicine, 13 (11), p. 100217.

ALCABES Phillip (2009). Dread. How fear and fantasy have fueled epidemics from the black death to avian flu. Philadelphia, Public Affairs.

ATLANI-DUAUlt Laëtitia, MERCIER Arnaud, ROUSSEAu Cécile, GUYOT Paul \& MOATTI Jean-Paul (2015). « Blood libel rebooted: Traditional scapegoats, online media, and the H1N1 epidemic », Culture, Medicine, and Psychiatry, 39, pp. 43-61.

BAGCCHI Sanjeet (2014). « Ebola haemorrhagic fever in West Africa », The Lancet Infectious Diseases, 14 (5), pp. 375.

BAGGINI Julian (2017). A short history of truth. Consolations for a post-truth world. London, Quercus. BASTUG Alive \& BODUR Hürrem (2015). « Ebola viral disease: What should be done to combat the epidemic in 2014? ", Turkish Journal of Medical Sciences, 45 (1), pp. 1-5.

BRAINARD Julii, HOOPER Lee, POND Katherine, EDMUNDS Kelly \& HUNTER Paul (2016). « Risk factors for transmission of Ebola or Marburg virus disease: a systematic review and meta-analysis ", International Journal of Epidemiology, 45 (1), pp. 102-116.

BROOM Alex \& BROOM Jennifer (2017). « Fear, duty and the moralities of care: The Ebola 2014 threat », Journal of Sociology, 51 (1), pp. 201-216.

BROUDOUX Évelyne (2017). « Autorité scientifique et épistémique à l'épreuve de la mesure des citations ", Études de communication, 1 (48), pp. 177-198.

CHANDLER Clare, FAIRHEAD James, KELLY Ann, LEACH Melissa, MARTINEAU Frederick, MOKUWA Esther, ... WILKINSON Annie (2015). « Ebola: limitations of correcting misinformation », The Lancet, 385 (9975), pp. 1275-1277.

CHEW Cynthia \& EYSENBACH Gunther (2010). «Pandemics in the age of Twitter: content analysis of tweets during the 2009 H1N1 outbreak », PLos ONE, 5 (11), p. 14118.

CHOWELL Gerardo \& VIBOUD Cécile (2015). « Controlling Ebola: key role of Ebola treatment centres », The Lancet Infectious Diseases, 15 (2), pp. 139-141. 
CLEATON Julie, Viboud Cécile, Simonsen Lone, hURTADo Ana \& CHOWELl Gerardo (2016).

«Characterizing Ebola transmission patterns based on Internet news reports », Clinical Infectious Diseases, 62 (1), pp. 24-31.

CURRAN Kathryn, GIBSON James, MARKE Dennis, CAULKER Victor, BOMEH John, REDD John, ... KILMARX Peter (2016). « Cluster of Ebola virus disease linked to a single funeral - Moyamba District, Sierra Leone, 2014 », Morbidity and Mortality Weekly Report, 65 (8), pp. 202-205. D'ANGELO Paul \& KUYPERS Jim (2010). Doing news framing analysis. Empirical and theoretical perspectives. London, Routledge.

DOUGLAS Mary (1992). Risk and blame. Essays in cultural theory. New York, Routledge.

Dozon Jean-Pierre (1991). « D’un tombeau l'autre », Cahiers d'études africaines, 121-122, pp. 135-157. DRY Sarah \& LEACH Melissa (2010). Epidemics. Science, governance and social justice. London, Earthscan.

ECHENBERG Myron (2011). Africa in the time of cholera. A history of pandemics from 1817 to the Present. New York, Cambridge University Press.

FABRE Gérard (1998). Épidémies et contagions. L'imaginaire du mal en Occident. Paris, Presses Universitaires de France.

FAIRHEAD James (2016). « Understanding social resistance to the Ebola response in the forest region of the Republic of Guinea: an anthropological perspective ", African Studies Review, 59 (3), pp. 7-31.

FARMER Paul (2006). Aids and accusations. Haiti and the geography of blame. Berkeley \& Los Angeles, University of California Press.

FAYE Ousmane, Bö̈LlE Pierre-Yves, HELEZE Emmanuel, FAYE Oumar, LOUCOUBAR Cheikh, MAGASSOUBA N'Faly, ... CAUCHEMEZ Simon (2015). « Chains of transmission and control of Ebola virus disease in Conakry, Guinea, in 2014: an observational study », The Lancet Infectious Diseases, 15 (3), pp. 320-326.

FOUCAUlT Michel (1978). Sécurité, territoire, population et Naissance de la biopolitique. Paris, Seuil.

FUNG Isaac Chun-Hai, DUKE Carmen, FINCH Kathryn, SNOOK Kassandra, TSENG Pei-Ling, HARNANDEZ Ana, ... TSE Zion Tsz Ho (2016). « Ebola Virus Disease and Social Media: A Systematic Review », American Journal of Infection, 44 (12), pp. 1660-1671.

GIRARD René (1982). Le Bouc émissaire. Paris, Grasset \& Fasquelle.

GREEN Andrew (2014). « West Africa struggles to contain Ebola outbreak », The Lancet, 383 (9924), p. 1196.

HELLER Jacob (2015). « Rumors and realities: making sense of HIV/AIDS conspiracy narratives and contemporary legends », American Journal of Public Health, 105 (1), pp. e43-e50.

HEWLETT Barry \& HEWLETT Bonnie (2008). Ebola, culture, and politics. The anthropology of an emerging disease. Belmont, Cengage Learning.

HYVÖNEN Ari-Elmeri (2018). « Careless speech: conceptualizing post-truth politics 1 », New Perspectives, 26 (3), pp. 31-55.

JALLOH Mohamed, ROBINSON Susan, CORKER Jamaica, LI Wenshu, IRWIN Kathleen, BARRY Alpha, ... BRUNNELL Rebecca (2017). « Knowledge, attitudes, and practices related to Ebola virus disease at 
the end of a national epidemic - Guinea, August 2015 », Morbidity and Mortality Weekly Report, 66 (41), pp. 1109-1115.

JOFFE Helene (2004). Risk and « the Other ». Cambridge, Cambridge University Press.

JOFFE Helene (2011). « Public apprehension of emerging infectious diseases: are changes afoot? », Public Understanding of Science, 20 (4), pp. 446-460.

KORO-LJUNGBERG Mirka, CARLSON David Lee \& MONTANA Anna (2019). «Productive forces of posttruth(s)? », Qualitative Inquiry, 25 (6), pp. 583-590.

KYRIAKIDES Christopher (2017). « Words don't come easy: Al Jazeera's migrant-refugee distinction and the European culture of (mis)trust », Current Sociology, 65 (7), pp. 933-952.

LAKOFF Andrew \& COLLIER Stephen J. (2008). Biosecurity interventions. Global health and security in question. New York, Columbia University Press.

LANDRY FAYE Sylvain (2015). «L'exceptionalité' d'Ebola et les 'réticences' populaires en GuinéeConakry. Réflexions à partir d'une approche d'anthropologie symétrique », Anthropologie et Santé, 11, doi: 10.4000/anthropologiesante.1796

LE MARCIS Frédéric (2015). « 'Traiter les corps comme des fagot'. Production sociale de l'indifférence en contexte Ebola (Guinée) ", Anthropologie et Santé, 11, doi : 10.4000/ anthropologiesante.1907

LEROY Frédéric, BRENGMAN Malaika, RYCKBOSCH Wouter \& SCHOLLIERS Peter (2018). « Meat in the posttruth era: mass media discourses on health and disease in the attention economy », Appetite, 125 ,pp. 345-355.

MARSHALL Katherine (2017). "Roles of religious actors in the West African Ebola response ", Development in Practice, 27 (5), pp. 622-633.

MARSHALL Katherine \& SMITH Sally (2015). « Religion and Ebola: learning from experience », The Lancet, 386 (10005), pp. e24-e25.

MAYOR Eric, EICHER Véronique, BANGERTER Andrian, GILLES Ingrid, CLÉMENCE Alain \& GREEN Eva (2013). « Dynamic social representations of the 2009 H1N1 pandemic: Shifting patterns of sense-making and blame », Public Understanding of Science, 22 (8), pp. 1011-1024.

MCNEILL William (1998). Plagues and peoples. New York, Anchor Books.

MITMAN Gregg (2014). « Bush meat: Ebola in a stew of fear », New England Journal of Medicine, 371, pp. 1763-1765.

MONSON Sarah (2017). « Ebola as African: American media discourses of panic and otherization », Africa Today, 63 (3), pp. 2-27.

MOREAU Nicolas, ROY Mélissa, WILSON Andrew \& ATLANI-DUAULT Laëtitia (2021). « 'Life is more important than football': comparative analysis of tweets and Facebook comments regarding the cancellation of the 2015 African Cup of Nations in Morocco ", International Review for the Sociology of Sport, 56 (2), pp. 252-275.

Moulin Anne Marie (2015). «L'anthropologie au défi de l'Ebola », Anthropologie et Santé, 11, doi : 10.4000/anthropologiesante.1954

MUDIMBE Valentin-Yves (1988). The invention of Africa. Gnosis, philosophy, and the order of knowledge. Bloomington, Indiana University Press.

MUDIMBE Valentin-Yves (1994). The idea of Africa. Bloomington, Indiana University Press. 
NAGPAL Sajan Jiy Singh, KARIMIANPOUR Ahmadreza, MUKHIJA Dhruvika \& MORAN Diwakar (2015). « Dissemination of « misleading » information on social media during the 2014 Ebola epidemic: An area of concern », American Journal of Infection Control, 13 (4), pp. 338-339.

NELKIN Dorothy \& GILMAN Sander (1991). «Placing blame for devastating disease », in Mack Arien (ed.) Time of plague: the history and social consequences of lethal epidemic disease, New York, New York University Press, pp. 39-56.

oDLuM Michelle \& Yoon Sunmoo (2015). « What can we learn about the Ebola outbreak from tweets? », American Journal of Infection Control, 44 (12), pp. 563-571.

OH Onook, AGRAWAL Manish \& RAGHAV Rao (2013). « Community intelligence and social media services: A rumor theoretic analysis of tweets during social crises », MIS Quarterly, 37 (2), pp. 407-426.

OMONZEJELE Peter (2014). «Ethical challenges posed by the Ebola virus epidemic in West Africa », Journal of Bioethical Inquiry, 11 (4), pp. 417-420.

ORCHARD Lisa, FULLWOOD Chris, MORRIS Neil \& GALBRAITH Niall (2015). « Investigating the Facebook experience through Q methodology: collective investment and a «borg » mentality », New Media \& Society, 17 (9), pp. 1547-1565.

ORGANISATION MONDIALE DE LA SANTÉ (2017). « Frequently asked questions on Ebola virus disease », Organisation mondiale de la santé, URL : http://www.who.int/csr/disease/ebola/faq-ebola/en/ ORGANISATION MONDIALE DE LA SANTÉ (2018). « Ebola virus disease. Key facts », (2018). Organisation mondiale de la santé, URL : http://www.who.int/news-room/fact-sheets/detail/ebola-virus-disease PANDEY Abhishek, ATKINS Katherine, MEDLOCK Jan, wENZEL Natasha, TOWNSEND Jeffrey, CHILDS James, ... GALVANI Alison (2014). « Strategies for containing Ebola in West Africa », Science, 346 (6212), pp. 991-995.

PASCUAL Cecilia (2017). « The cholera epidemic as condenser of meanings: urban cultures, clinical narratives, and hygiene policies in Rosario, Argentina, 1886-1887 », Historia, Ciencias, SaudeManguinhos, 24 (2), pp. 295-311.

PAUL Katharina \& HADDAD Christian (2019). « Beyond evidence versus truthiness: Toward a symmetrical approach to knowledge and ignorance in policy studies », Policy Sciences, 52 (2), pp. 299-314.

PICARD Robert \& VIAL Antoine (2012). « De l'information du patient à la démocratie sanitaire : enjeux et conditions d'un usage efficient des technologies ", Ministère de l'économie et des finances de la République française.

PIOT Peter, MUYEMBE Jean-Jacques \& EDMUNDS John (2014). « Ebola in West Africa : From disease outbreak to humanitarian crisis », The Lancet Infectious Diseases, 14 (11), pp. 1034-1035.

PROZOROV Sergei (2019). « Why is there truth? Foucault in the age of post-truth politics ", Constellations, 26 (1), pp. 18-30.

RAVI Sanjana \& GAULDIN Eric (2014). « Sociocultural dimensions of the Ebola virus disease outbreak in Liberia », Biosecurity and Bioterrorism: Biodefense Strategy, Practice, and Science, 12 (6), pp. 301-305. RENARD Jean-Bruno (2011). « Les rumeurs et Internet », Contemporanea, 9 (3), pp. 475-483. RICHARDS Paul (2016). Ebola. How a people's science helped end an epidemic. London, Zed. 
ROY Mélissa, MOREAu Nicolas, ROUSSEAu Cécile, MERCIER Arnaud, WILSON Andrew \& ATLANI-DUAULT Laëtitia (sous presse). « Constructing Ebola martyrs, warriors, and saviors: Online heroization in a context of risk and unease », Health, Risk \& Society.

ROY Mélissa, MOREAu Nicolas, ROUSSEAu Cécile, MERCIER Arnaud, wiLSON Andrew \& ATLANI-DUAULT Laëtitia (2020). « Ebola and localized blame on social media : Analysis of Twitter and Facebook conversations during the 2014-2015 Ebola epidemic », Culture, Medicine, and Psychiatry, 44 (1), pp. 56-79.

SIMON Justine \& TOULLEC Bénédicte (2017). « Identifier et analyser les discours d'escorte sur Twitter. L'infuence des discours d'accompagnement sur le partage social », in Wigham Ciara R. \& Ledegen Gudrun (eds.) Corpus de communication médiée par les réseaux : construction, structuration, analyse, Paris, L'Harmattan, pp. 140-152.

SINHA Michael \& PARMET Wendy (2016). « The perils of panic: Ebola, HIV, and the intersection of global health and law ", American Journal of Law and Medicine, 42 (2,3), pp. 223-255.

THACKERAY Rosemary, NEIGER Brad, SMITH Amanda \& VAN WAGENEN Sarah (2012). « Adoption and use of social media among public health departments », BMC Public Health, 12, p. 242.

VANCE Karl, HoWE William \& DELLAVALLE Robert P. (2009). « Social Internet sites as a source of public health information », Dermatologic Clinics, 27 (2), pp. 133-136.

VICTORY Kerton, CORONADO Fátima, IFONO Sâa, SOROPOGUI Therese \& DAHL Benjamin (2015). « Ebola transmission linked to a single traditional funeral ceremony - Kissidougou, Guinea, December, 2014-January 2015 », Morbidity and Mortality Weekly Report, 64 (14), pp. 386-388.

WAGNER-EGGER Pascal, BANGERTER Andrian, GILLES Ingrid, GREEN Eva, RIGAUD David, KRINGS Franciska, ... CLÉMENCE Alain (2011). « Lay perceptions of collectives at the outbreak of the H1N1 epidemic: heroes, villains and victims », Public Understanding of Science, 20 (4), pp. 461-476.

WALD Priscilla (2008). Contagious. Cultures, carriers, and the outbreak narrative. Durham, Duke University Press.

WARD Jeremy, GUILLE-ESCURET Paul \& ALAPETITE Clément (2019). « Les « antivaccins », figure de l'anti-science ", Médecine \& Hygiène, 43 (2), pp. 221-251.

WONG Roger, HARRIS Jenine, STAUB Mackenzie \& BERNHARDT Jay (2017). « Local health departments tweeting about Ebola: characteristics and messaging ", Journal of Public Health Management and Practice, 23 (2), pp. e16-e24.

WOTEM SOMPARÉ Abdoulaye (2017). «La politique et les pratiques de santé en Guinée à l'épreuve de l'épidémie d'Ebola : le cas de la ville de Conakry », Lien social et Politiques, 78 , pp. 193-210.

YUYA Sugawara, NARIMATSU Hiroto, HOZAWA Atsushi, SHAO Li, OTANI Katsumi \& FUKAO Akira (2012).

«Cancer patients on Twitter: a novel patient community on social media », BMC Research Notes, 5, pp. 699.

\section{NOTES}

1. Le concept de politique biosécuritaire renvoie à un régime de gouvernance qui veut protéger la vie humaine (Foucault, 1978) en "sécurisant la santé » (Lakoff et Collier, 2008). Elle se concrétise par le déploiement d'interventions qui répondent aux menaces microbiennes (réelles ou perçues, actuelles ou anticipées), et qui s'articulent par la conjonction de pratiques de sécurisation (fermetures, interdictions, contrôle de la 
circulation, interventions policières ou militaires) et de techniques de sanitarisation (désinfection, équipement de protection personnelle) (Wotem Somparé, 2017).

2. Commentaire Facebook à l'article "Courage, death and survival: On the front lines of the Ebola outbreak », Globe and Mail, 9 août 2014, notre traduction.

3. Commentaire Facebook à l'article "Some Families Affected by Ebola in Liberia Pay Bribes to Keep the Bodies ", Yahoo News, 17 octobre 2014, notre traduction.

4. Tweet, 17 août 2014.

5. Tweet, 9 décembre 2014, notre traduction.

6. Tweet, 16 octobre 2014.

7. Commentaire Facebook à l'article " 'Prophet' under fire for Nigeria building collapse ", Globe and Mail, 18 septembre 2014, notre traduction

8. Commentaire Facebook à l'article " Ann Coulter, Donald Trump Ebola Comments Prompt Christian Backlash », Huffington Post, 11 août 2014, notre traduction.

9. Commentaire Facebook à l'article « 'Prophet' under fire for Nigeria building collapse ", Globe and Mail, 18 septembre 2014, notre traduction.

10. Commentaire Facebook à l'article « Report : Ebola Outbreak Probably Started with 2 Year Old in Guinea », CNN, 11 août 2014, notre traduction.

11. Tweet, 7 novembre 2014, notre traduction

12. Tweet, 7 novembre 2014

13. Tweet, 9 novembre 2014 , notre traduction

14. Tweet, 5 août 2014, nous soulignons

15. Commentaire Facebook à l'article "Nigeria: un médecin contracte l'Ebola ", Le Figaro, 5 août 2014.

16. Commentaire Facebook à l'article "Crisis of political leadership ", The Perspective, 14 novembre 2014, notre traduction.

17. Tweet, 30 octobre 2014.

18. Commentaire Facebook à l'article «Ebola Now National Emergency - Health Minister ", Vanguard Nigeria, 7 août 2014, notre traduction

19. Commentaire Facebook à l'article « Facts : Ebola Virus Disease », Vandgard, 30 juillet 2014, notre traduction

20. Commentaire Facebook à l'article «Thomas Eric Duncan, Dallas Ebola Patient, Now in Critical Condition », Huffington Post, 7 octobre 2014, notre traduction.

21. Commentaire Facebook à l'article « Ebola Alert! FG stops transportation of corpses into Nigeria ", Vanguard, 2 août 2014, notre traduction, nous soulignons.

22. Commentaire Facebook à l'article "Report: 5 Year Old Hospitalized in NYC With Possible Ebola Symptoms », CNN, 27 octobre 2014, notre traduction, nous soulignons. 


\section{RÉSUMÉS}

Les discours accusatoires sont une dynamique récurrente dans l'histoire des épidémies. Si les études sur le sujet ont tendance à recenser des figures du blâme et montrer en quoi leur accusation renforce des enjeux sociaux préexistants, cette recherche ajoute une perspective complémentaire à ce corpus d'écrits en analysant le travail rhétorique de la production du blâme. À partir du concept de cadres, elle s'intéresse aux logiques argumentatives sous-jacentes aux accusations déployées dans le débat thanatologique lors de l'épidémie Ebola 2013-2016. Notre analyse des commentaires sur Facebook et Twitter indique que cinq cadres accusatoires justifient le déploiement de discours entourant les pratiques funéraires traditionnelles: l'opposition religion - science; l'inaction et l'irresponsabilité; les cultures locales/africaines; la corruption; et la pauvreté. Ces cadres sont appuyés par les autorités sanitaires et les médias, dont les discours sont remis en circulation par les usagers de médias sociaux afin de justifier leurs arguments accusatoires. L'analyse montre que l'usage de ces schèmes et le choix de figures du blâme privilégiées varient selon l'ancrage géographique des usagers de médias sociaux. Une lecture transversale des résultats indique que les cadres accusatoires mobilisent l'« irrationalité » des accusés, et qu'ils ciblent des figures récurrentes, notamment le continent africain et ses habitants. Nous émettons l'hypothèse que ces discours réarticulent des normes et craintes qui vont au-delà de la peur de la maladie et de l'Autre. Ils sont aussi ancrés dans des frustrations préexistantes et réarticulent une préoccupation contemporaine à l'égard de la "désinformation». En guise de conclusion, nous suggérons des pistes de réflexion pour les stratégies de communication en ligne.

Accusatory discourses are a recurring dynamic in the history of epidemics. Previous studies tend to explore figures of blame and show how their accusation reinforces social issues. This research adds a complementary perspective to this body of work by analysing the rhetorical work associated with the production of blame. Drawing on the concept of frames, it is interested in the argumentative logics underlying the accusations circulating in the thanatological debate during the 2013-2016 Ebola epidemic. Our analysis of comments published on Facebook and Twitter indicates that five accusatory frames justified the use of accusatory discourses surrounding traditional funeral practices: a religion-science opposition; inaction and irresponsibility; local/ African cultures; corruption; and poverty. These frames are sustained by health authorities and the media, because social media users share and rely on both institutions' discourses to justify their accusatory arguments. Our analysis shows that the use of these frames and the chosen figures of blame vary according to social media users' geographical ties. A transversal reading of the results indicates that the accusatory frames argue the "irrationality" of the accused, and that they target recurring figures of blame, namely the African continent and its inhabitants. We postulate that these discourses rearticulate norms and preoccupations that are beyond the fear of the disease and of the Other. They are also situated in pre-existing frustrations and rearticulate a contemporary preoccupation relating to "disinformation". In conclusion, we present points for reflection to improve online communication strategies.

\section{INDEX}

Mots-clés : Accusation, Cadre, Ebola, Funérailles, Médias sociaux

Keywords : Accusation, Frame, Ebola, Funeral, Social media 
AUTEUR

MÉLISSA ROY

Université d'Ottawa, École de service social 